\title{
Transition readiness assessment: The importance of the adolescent perspective
}

There is concern that medical complications increase following the transfer from pediatric to adult-centered transplant care, perhaps in part due to medication non-adherence. ${ }^{1,2}$ Thus, there is a need to develop strategies to assess an adolescent/young adult's (AYA's) readiness to move to an adult-centered clinic. "Transition" has been described as an active process that addresses the medical, psychosocial, and educational/vocational needs of adolescents as they prepare to move from child- to adult-centered health care, while "transfer" refers to the change in the location where care is provided. ${ }^{4}$ Moreover, transition also relates to the gradual transition of responsibility from parent to the AYA. ${ }^{5,6}$ Transition readiness can be viewed as an AYA's ability to begin and complete the transition process with parent/family and provider support. ${ }^{7}$

There are various potential barriers to transitioning and ultimately transferring care. These barriers arise at the level of the patient, parent/family, and the pediatric and adult provider. Thus, there is growing support for a multidimensional assessment of patients, parents, and providers, as transition is more than the individual's age, health-related knowledge, and skills. ${ }^{8,9}$ Ideally, the assessment of transition-related skills would be conducted using well-validated measures in the context of standard clinical care. Although there is not an accepted "gold standard" assessment tool, there is an increased body of literature supporting measures that assess domains of self-management and transition readiness. ${ }^{6,9-14}$ The Readiness for Transition Questionnaire (RTQ) is a unique measurement system that allows for the assessment of transition readiness perceptions, adolescent responsibility for healthcare behavior, and parental involvement from the perspective of the adolescent, parent/caregiver, and healthcare provider. ${ }^{10,15}$

The ability to assess transition readiness from multiple perspectives is critical, as there are often discrepancies in the reports of AYAs, parents, and their providers. In several studies of AYA transplant recipients, there were significant differences between parent and AYA liver transplant recipients' perceptions of healthcare responsibility, whereas AYAs perceive they have more responsibility for health management tasks than their parents/providers perceive them to have. ${ }^{15-17}$ Discrepancies across respondents suggest that parents/ providers are either underestimating the AYA's ability to self-manage their healthcare needs, or perhaps the AYA are overestimating their independence in healthcare self-management. Alternatively, the "truth" may lie somewhere in between.

While discrepancies between respondents are common, they are not universal. In a study of AYA liver transplant recipients and their parents, there was general consensus between respondents on a measure assessing allocation of responsibility for health-related tasks. This may be due to the fact that this measure assesses observable behaviors, which may be more objective than subjective feelings of "readiness." Nonetheless, when discrepancies are noted, it is difficult to identify whether differences are due to AYA overestimation of their abilities, parent under-appreciation of their AYAs abilities, or somewhere in the middle. As parents are often hesitant to shift responsibility for health management tasks to their AYA, it is often challenging to identify what tasks the adolescent is capable of managing. ${ }^{15}$ Several factors may account for the parent-AYA discrepancy in perceived responsibility, including the difficulty for parents to reliably report on their AYA's internal state or self-management behaviors. It may also be that parents are engaged in tasks that their AYA does not notice, such as refilling prescriptions or scheduling clinic visits. The AYA may perceive that these activities occur somewhat automatically. Conversely, some parents prematurely transfer responsibility before their adolescent is adequately prepared to independently manage healthcare-related tasks, as they perceive their AYA to be "old enough" to take over responsibility. Thus, it is important to assess transition readiness from multiple perspectives. ${ }^{6,15,16}$

In the current study by Guitierrez-Colina et al., ${ }^{18}$ only caregiver (parent) report of transition readiness was used, due to time restrictions and logistical limitations. These barriers are very common in clinical settings and underscore the importance of translational research and quality improvement initiatives to identify best practices for incorporating assessment of adolescent health behaviors in routine clinical practice. The authors aptly noted that parents often underestimate AYA abilities, particularly with respect to transition readiness and adherence. Thus, they recommend that future research is needed to understand at which point parents/caregivers should transition responsibility for healthcare management tasks to their adolescents. This study highlights the importance of making decisions based on developmental level and skills, particularly executive functioning (EF) skills. Deficits in EF likely will interfere with behaviors necessary for optimal adherence and transition of health care. Guitierrez-Colina and colleagues note that increased parental involvement may serve as a protective factor among AYAs who are at risk for medication nonadherence, perhaps due to poorer EF. ${ }^{18}$ Indeed, AYAs with deficits in EF skills are likely to require increased parental monitoring and support throughout the transition process, despite seeming "old enough" based on chronological age.

Overall, the assessment and promotion of transition readiness and successful transfer to adult transplant care remains a complex and understudied area. The focus on EF skills in transition readiness is an important contribution and may lead to targeted interventions for 
AYAs who have deficits in this area of cognitive functioning. As such, it is worth considering routine screening of EF skills to identify children and young adolescents who are at increased risk. Deficits in EF skills may also contribute to discrepancies between reports obtained from AYAs, parents, and providers. Future research should continue to obtain multi-informant assessments to build on the identification of protective factors and modifiable barriers in order to promote optimal long-term, positive outcomes among pediatric transplant recipients.

Emily M. Fredericks

Division of Pediatric Psychology, Child Health Evaluation and Research (CHEAR) Center, University of Michigan Medical School and C.S. Mott Children's Hospital, Ann Arbor, MI, USA

\section{REFERENCES}

1. Watson AR. Problems and pitfalls of transition from paediatric to adult renal care. Pediatric Nephrol. 2005;20:113-117.

2. Foster BJ. Heightened graft failure risk during emerging adulthood and transition to adult care. Pediatr Nephrol. 2015;30:567-576.

3. Bell LE, Bartosh SM, Davis CL, et al. Adolescent transition to adult care in solid organ transplantation: a consensus conference report. Am J Transplant. 2008;8:2230-2242.

4. Sawyer SM, Blair S, Bowes G. Chronic illness in adolescents: transfer or transition to adult services? J Paediatr Child Health. 1997;33: 88-90.

5. Bilhartz JL, Lopez MJ, Magee JC, Shieck VL, Eder SJ, Fredericks EM. Assessing allocation of responsibility for health management in pediatric liver transplant recipients. Pediatr Transplant. 2015;19:538-546.

6. Fredericks EM, Dore-Stites D, Well A, et al. Assessment of transition readiness skills and adherence in pediatric liver transplant recipients. Pediatr Transplant. 2010;14:944-953.

7. de Silva PS, Fishman LN. Transition of the patient with IBD from pediatric to adult care-an assessment of current evidence. Inflamm Bowel Dis. 2014;20:1458-1464.
8. Annunziato RA, Parkar S, Dugan CA, et al. Brief report: deficits in health care management skills among adolescent and young adult liver transplant recipients transitioning to adult care settings. J Pediatr Psychol. 2011;36:155-159.

9. Reed-Knight B, Blount RL, Gilleland J. The transition of health care responsibility from parents to youth diagnosed with chronic illness: a developmental systems perspective. Families Syst Health. 2014;32:219-234.

10. Gilleland J, Amaral S, Mee L, Blount R. Getting ready to leave: transition readiness in adolescent kidney transplant recipients. J Pediatr Psychol. 2012;37:85-96.

11. Ferris ME, Harward DH, Bickford $\mathrm{K}$, et al. A clinical tool to measure the components of health-care transition from pediatric care to adult care: the UNC TR(x)ANSITION scale. Ren Fail. 2012;34:744-753.

12. Sawicki GS, Lukens-Bull K, Yin $X$, et al. Measuring the transition readiness of youth with special healthcare needs: validation of the TRAQ Transition Readiness Assessment Questionnaire. ediatr Psychol. 2011;36:160-171.

13. Zhang LF, Ho JS, Kennedy SE. A systematic review of the psychometric properties of transition readiness assessment tools in adolescents with chronic disease. BMC Pediatr. 2014;14:4.

14. Schwartz LA, Daniel LC, Brumley LD, Barakat LP, Wesley KM, Tuchman LK. Measures of readiness to transition to adult health care for youth with chronic physical health conditions: a systematic review and recommendations for measurement testing and development. $J$ Pediatr Psychol. 2014;39:588-601.

15. Marchak JG, Reed-Knight B, Amaral S, Mee L, Blount RL. Providers' assessment of transition readiness among adolescent and young adult kidney transplant recipients. Pediatr Transplant. 2015;19:849-857.

16. Fredericks EM, Dore-Stites D, Lopez MJ, et al. Transition of pediatric liver transplant recipients to adult care: patient and parent perspectives. Pediatr Transplant. 2011;15:414-424.

17. Kullgren KA, Hmiel SP, Gevers A. Measuring family management of transplant tasks: the transplant responsibility questionnaire. Pediatr Transplant. 2013;17:307-314

18. Gutierrez-Colina AM, Reed-Knight B, Eaton C, et al. Transition readiness, adolescent responsibility and executive functioning among pediatric transplant recipients. Pediatr Transplant. 2017;21:e12898; https://doi.org/10.1111/petr.12898. 